\title{
Diagnostik von berufsbedingten Typ-I-Allergien - Vergleich von Haut-Pricktestlösungen verschiedener Hersteller für ausgewählte Berufsallergene
}

\author{
Diagnostics of Occupational Type I Allergies - Comparison of Skin Prick Test \\ Solutions from Different Manufacturers for Selected Occupational Allergens
}

Autoren

Institut
V. van Kampen, M. Raulf-Heimsoth, I. Sander, R. Merget und die Stad 1-Gruppe

Institut für Prävention und Arbeitsmedizin der Deutschen Gesetzlichen Unfallversicherung, Institut der Ruhr-Universität Bochum (IPA) (Direktor: T. Brüning) eingereicht 16.11.2009

akzeptiert 28.1.2010

\section{Bibliografie}

Dol http://dx.doi.org/ 10.1055/s-0029-1243972 Pneumologie 2010; 64: 271-277 @ Georg Thieme Verlag KG Stuttgart · New York ISSN 0934-8387

\section{Korrespondenzadresse} Dr. rer. nat. Vera van Kampen IPA (vormals BGFA) Bürkle-de-la-Camp-Platz 1 44789 Bochum kampen@ipa-dguv.de

\section{Zusammenfassung \\ $\nabla$}

Hintergrund: Das Ergebnis des Haut-Pricktests (HT) hängt von der Qualität der HT-Lösung ab. Ziel der Untersuchung war der Vergleich von HTLösungen verschiedener Hersteller für ausgewählte Berufsallergene.

Patienten: Alle Patienten klagten über arbeitsplatzbezogene allergische Beschwerden wie Rhinits, Konjunktivitis oder asthmatische Beschwerden.

Methoden: 125 Bäcker bzw. 21 Naturgummilatex-Exponierte wurden im HT mit vier Mehlbzw. zwei Naturgummilatex-HT-Lösungen untersucht. Anhand der maximalen Testeffizienz (Gold-Standard: spezifisches IgE) wurde der optimale Cut-off ermittelt. Bei diesem Cut-off wurden Sensitivität und Spezifität der Mehl- und Naturgummilatex-HT-Lösungen bestimmt. Zusätzlich wurden fünf HT-Lösungen für Tyrophagus putrescentiae (Tp) bei zwei Landwirten getestet. Sämtliche HT-Lösungen wurden in vitro bzgl. Proteinund Antigengehalt analysiert.

Ergebnisse: Die In-vitro-Analyse ergab für alle HT-Lösungen herstellerabhängige Unterschiede. Der optimale Cut-off für die Mehl- und Naturgummilatex-HT-Lösungen lag bei einer Quaddelgröße $\geq 1,5 \mathrm{~mm}$. Die Sensitivitäten der Mehl-HTLösungen betrugen 38-95\%, die Spezifitäten 77-98\%. Beide Naturgummilatex-HT-Lösungen ergaben eine Sensitivität von $100 \%$ bei einer Spezifität von $92 \%$. Die verschiedenen Tp-HT-Lösungen lieferten diskrepante HT-Ergebnisse.

Schlussfolgerung: Während die beiden Naturgummilatex-HT-Lösungen eine gleich gute Qualität aufwiesen, zeigten die Mehl- und Tp-HT-Lösungen deutliche Unterschiede. HT-Lösungen mit höheren Protein- bzw. Antigengehalten wiesen in der Regel eine bessere Qualität auf.

\section{Abstract \\ $\nabla$}

Background: Reactivity in the skin prick test (SPT) is related to the potency of the SPT solution used. The aim of this study was to compare SPT solutions from different manufacturers for selected occupational allergens.

Patients: All patients suffered from work-related allergic complaints like rhinitis, conjunctivis or asthmatic complaints.

Methods: 125 bakers and 21 subjects exposed to natural rubber latex (NRL) were examined with four flour SPT solutions and two NRL SPT solutions, respectively. By means of the maximum test efficiency (gold standard: specific IgE), the optimal cut-off was determined. Using this cut-off, sensitivities and specificities of the flour and NRL SPT solutions were calculated. Additionally, five SPT solutions for Tyrophagus putrescentiae (Tp) were tested in two farmers. All SPT solutions were analysed in-vitro for protein and antigen content.

Results: In-vitro analyses resulted in differences for all SPT solutions depending on the manufacturer. The optimal cut-off for the flour and NRL SPT solutions was a wheal $\geq 1.5 \mathrm{~mm}$. Sensitivities of flour SPT solutions ranged from $38 \%$ to $95 \%$, specificities from $77 \%$ to $98 \%$. Both NRL SPT solutions reached a sensitivity of $100 \%$ and a specificity of 92\%. SPT results with the different Tp SPT solutions were variable.

Conclusion: While both NRL SPT solutions exhibited a fairly good quality, the flour and Tp SPT solutions showed differences between manufacturers. As a rule solutions with higher protein and antigen contents gave better results. 


\section{Einleitung}

$\nabla$

Die eindeutige Diagnostik von berufsbedingten IgE-vermittelten Allergien ist sowohl bei Berufskrankheiten(BK)-Feststellungsverfahren als auch für Untersuchungen im Rahmen von betrieblichen Präventionsmaßnahmen sowie für wissenschaftliche Studien von großer Bedeutung. Vor allem der Haut-Pricktest (HT) ist ein schnelles, preisgünstiges und - bei standardisiertem methodischen Vorgehen mit validierten und standardisierten Extrakten - auch ein sensitives Verfahren zum Nachweis von Sensibilisierungen vom Soforttyp.

Allerdings konnte schon in einer früheren Arbeit gezeigt werden, dass die für die Diagnostik des Bäckerasthmas unverzichtbaren kommerziellen Mehl-HT-Lösungen in Abhängigkeit vom Hersteller in ihrer Qualität stark divergieren [1]. Dies ist umso erstaunlicher, als das Bäckerasthma in Deutschland keine seltene Erkrankung darstellt. Von 587 im Jahr 2008 bestätigten Fällen einer berufsbedingten obstruktiven allergischen Atemwegserkrankung (BK 4301) waren 372 (63\%) auf Mehle und Mehlprodukte zurückzuführen [2]. Ein weiteres relevantes Berufsallergen stellt das Naturgummilatex (im Folgenden nur noch als Latex bezeichnet) dar. Nachdem die Zahl der dadurch hervorgerufenen allergischen Berufskrankheiten vor allem in den 1990er-Jahren im Gesundheitswesen im Rahmen der HIV-Epidemie sehr stark angestiegen war, sank sie ab 1998 aufgrund von Präventionsmaßnahmen wieder deutlich ab. Im Jahr 2008 waren noch 18 (3\%) bestätigte BK 4301-Fälle in Deutschland auf Latex zurückzuführen [2]. Da es sich jedoch bei Latex um ein sehr potentes Allergen handelt, das bei entsprechend Sensibilisierten häufig zu schweren allergischen Reaktionen führt, ist eine zuverlässige Allergiediagnostik bei gegenüber Latex exponierten Personen mit allergischen Symptomen weiterhin von Bedeutung.

Ziel der Studie war es daher, die Qualität weiterer, z. T. selbst hergestellter Mehl-HT-Lösungen sowie verschiedener kommerzieller Latex-HT-Lösungen durch vergleichende Pricktestung und durch verschiedene In-vitro-Methoden zu bewerten. Darüber hinaus sollte beispielhaft für die Vorratsmilbe Tyrophagus putrescentiae überprüft werden, ob auch bei anderen Berufsallergenen mit Unterschieden in der Qualität der HT-Lösungen zu rechnen ist. Vorratsmilben spielen als berufliche Allergene insbesondere im landwirtschaftlichen Bereich und dort vor allem bei der Getreidelagerung eine Rolle [3].

\section{Methoden \\ $\nabla$}

\section{Studiendesign}

Um verschiedene HT-Lösungen für Mehle und Latex in möglichst großen Kollektiven zu überprüfen, haben sich sieben allergologisch gutachterlich tätige Zentren ( 6 deutsche, 1 polnisches) zu einem Verbund (Stad 1: Standardallergiediagnostik Typ 1) zusammengeschlossen. Gemeinsam einigten sich alle Beteiligten unter Berücksichtigung der europäischen Positionspapiere [4,5] auf einen Standard-Pricktest. Zudem wurden ein einheitlicher Fragebogen und ein Erfassungsbogen für ggf. vorliegende Provokationstestergebnisse entwickelt. Die Verbundpartner erhielten definierte HT-Lösungen zur Diagnose der sogenannten Bäckerbeziehungsweise Latexallergie, mit denen die Patienten im Rahmen von Begutachtungen getestet wurden.

Zusätzlich wurden im IPA verschiedene HT-Lösungen für die Vorratsmilbe Tyrophagus putrescentiae im titrierten Pricktest bei zwei sensibilisierten Probanden eingesetzt.
Alle im Rahmen der Studie verwendeten HT-Lösungen wurden in vitro hinsichtlich Protein- und Antigengehalt analysiert.

\section{Patienten}

Alle untersuchten Probanden klagten über arbeitsplatzbezogene allergische Beschwerden wie Rhinitis, Konjunktivitis oder asthmatische Beschwerden. Sämtliche Untersuchungen wurden im Rahmen von Begutachtungen durchgeführt. Für die Studie lag ein positives Votum der Ethik-Kommission der Ruhr-Universität Bochum vor und alle Patienten haben vor der Pricktestung ihr Einverständnis schriftlich bestätigt.

\section{Bäcker}

88 Bäcker (70\%) gaben asthmatische Beschwerden an, 80 davon in Kombination mit Rhinitis. 32 Bäcker (26\%) klagten über Rhinitis, jedoch ohne asthmatische Symptome. Das mittlere Alter lag bei $41 \pm 13$ Jahren, 44 (35\%) rauchten aktuell, 20 (16\%) waren Exraucher. Nach den Ergebnissen des HT mit ubiquitären Allergenen waren 61 Patienten (49\%) atopisch. Zum Zeitpunkt der Untersuchung waren noch 44 Bäcker (35\%) in ihrem Beruf tätig. Die mittlere Zeit seit Tätigkeitsaufgabe lag bei 4,3 \pm 3,8 Jahren.

\section{Latex-Exponierte}

Von den 21 gegenüber Latex exponierten Personen kamen ursprünglich 19 (91\%) aus dem Gesundheitswesen. Eine Person war in der Vulkanisierung, eine weitere in der kunststoffverarbeitenden Industrie tätig. Insgesamt 14 Personen (67\%) klagten über asthmatische Beschwerden, 12 davon in Kombination mit Rhinitis. In 2 Fällen wurde Rhinitis (10\%) ohne asthmatische Symptome angegeben. Das mittlere Alter lag bei $43 \pm 9$ Jahren, jeweils 4 Personen (19\%) waren Raucher bzw. Exraucher. Bei zehn Patienten (48\%) handelte es sich um Atopiker. Zum Zeitpunkt der Untersuchung waren noch 9 Latex-Exponierte (43\%) in ihrem Beruf tätig. Die mittlere Zeit seit Tätigkeitsaufgabe betrug 3,1 $\pm 3,3$ Jahre.

\section{Landwirte}

Beide Landwirte (43 bzw. 56 Jahre) klagten über berufsbezogene asthmatische Beschwerden und waren zum Zeitpunkt der Untersuchung noch aktuell tätig.

\section{Spezifische IgE-Antikörper (slgE)}

Die Quantifizierung der spezifischen IgE-Antikörper (sIgE) im Serum der Patienten erfolgte zentral im IPA mittels CAP-FEIA (Phadia, Freiburg). CAP-Werte $\geq 0,35 \mathrm{kU} / \mathrm{L}$ (CAP-Klasse $\geq 1$ ) galten als positiv.

\section{Haut-Pricktest (HT)}

Die vergleichenden HT-Untersuchungen im Rahmen des Stad 1Verbundprojektes wurden bei 125 Bäckern bzw. 21 Latex-Exponierten mit Weizen- und Roggenmehl-HT-Lösungen der Firmen Allergopharma (Reinbek), Bencard (München) und Hal (Düsseldorf) bzw. mit Latex-HT-Lösungen der Firmen Allergopharma und Stallergènes (Kamp-Lintfort) durchgeführt. Das IPA lieferte zusätzlich selbst hergestellte HT-Lösungen mit bekanntem Allergenprofil für Weizen- und Roggenmehl [6]. Um Aussagen über den Atopiestatus des jeweiligen Patienten treffen zu können, wurden zusätzlich vier kommerzielle Umweltallergene in das Testpanel aufgenommen: Graspollenmischung, Birke, Katzenepithelien und Hausstaubmilbe (Dermatophagoides pteronyssinus; alle Allergopharma). Als Atopiker galten die Personen, die mit 
mindestens einem der ubiquitären Allergene im HT mit einer Quaddel von $3 \mathrm{~mm}$ oder größer reagierten.

Nicht im Rahmen des Verbundprojektes, sondern nur in einem Zentrum (IPA), wurden fünf kommerzielle HT-Lösungen für die Vorratsmilbe Tyrophagus putrescentiae (Allerbio (über Themocare) Mönchengladbach; Allergopharma; ALK-Abelló, Wedel; Lofarma, Mailand, Italien; Stallergènes) in fünf verschiedenen Verdünnungen (jeweils $1: 4$; unverdünnt bis $1: 1024$ ) bei zwei Landwirten mit entsprechenden berufsbedingten allergischen Beschwerden eingesetzt.

Sämtliche HTs wurden ausschließlich im Rahmen von Begutachtungen nach einem exakt festgelegten Schema durchgeführt. Die HT-Lösungen wurden zunächst in Doppelbestimmung gegenläufig auf beide unbehandelte Unterarme aufgebracht, bevor die Haut mittels einer Stahllanzette (ALK-Abelló) durchstochen wurde. Histamin (10 mg/ml) bzw. Kochsalzlösung dienten als Positivbzw. Negativkontrolle. Nach 15 Minuten wurden die HT-Lösungen mit Alkohol abgewischt, die Quaddelumrisse mit Kugelschreiber umfahren und mit Hilfe von durchsichtigem Klebeband auf ein weißes Blatt Papier übertragen. Die Ausmessung der Quaddelgrößen (Mittelwert aus längstem und mittig dazu senkrecht stehendem Quaddeldurchmesser in Millimeter) und Bewertung aller HTs erfolgten zentral im IPA.

Für die Auswertung im Rahmen des Stad 1-Verbundprojektes (Mehl- und Latex-HT-Lösungen) wurde jeweils die Gesamtzahl aller Tests berücksichtigt, d.h. zwei Tests pro Proband und Allergen. Der jeweils optimale Cut-off-Wert wurde anhand der maximalen Testeffizienz ermittelt.

\section{Spezifischer Provokationstest}

Entsprechend der Anamnese und der Entscheidung des jeweiligen Gutachters wurde bei einigen Patienten ein Provokationstest durchgeführt. Von 125 Bäckern unterzogen sich 95 einem spezifischen Provokationstest mit Roggenmehl (bronchial mittels kommerziellem wässrigen Allergenextrakt: $n=24$; nasal mittels kommerziellem wässrigen Allergenextrakt: $\mathrm{n}=8$; arbeitsplatzbezogener Inhalationstest mit nativem Mehl: $n=63)$ [7]. Zehn der 21 Latex-Exponierten wurden mit Latex (Tragetest mit latexallergenhaltigem Handschuh) und beide Landwirte bronchial mit Tyrophagus putrescentiae (vernebelter wässriger Extrakt, ALKAbelló) provoziert.

Allergen-induzierte Veränderungen der Lungenfunktion wurden mittels Bodyplethysmografie gemessen. Als positiv galten eine Verdopplung des Atemwegswiderstandes (sRt) bei gleichzeitigem Anstieg auf $\geq 2.0 \mathrm{kPa}^{*} \mathrm{~s}$ und ein Abfall der Einsekundenkapazität $\left(\mathrm{FEV}_{1}\right)$ um $\geq 20 \%$.

Der nasale Atemwegswiderstand wurde mittels anteriorer Rhinomanometrie bestimmt. Bei einer Zunahme des nasalen Widerstandes und bei Abnahme des nasalen Flusses um $40 \%$ oder bei anhaltendem Fließschnupfen galt die Testung als positiv.

\section{Protein- und Antigenbestimmungen}

Die Bestimmung des Proteingehaltes der HT-Lösungen erfolgte mittels Bradford-Test unter Verwendung von Rinderserumalbumin als Standard [8].

Der Antigengehalt der HT-Lösungen für Weizenmehl [9], Roggenmehl [10] bzw. für Tyrophagus putrescentiae [11] wurde mittels spezifischer Sandwich Enzyme-Linked Immunosorbent Assays (ELISA), wie beschrieben, quantifiziert. Alle drei Sandwich ELISAs basieren auf polyklonalen Antikörpern gegen das gesamte lösliche Antigenspektrum der jeweiligen Ausgangssubstanz.
Der Gesamt-Latexallergengehalt beider Latex-HT-Lösungen wurde mittels CAP-Inhibitionstest ermittelt [12].

\section{Natriumdodecylsulfat (SDS)-Gelelektrophorese}

$10 \mu \mathrm{L}$ jeder HT-Lösung wurden in Fertiggelen (NuPAGE ${ }^{\circledR}$ Bis-Tris gel, Invitrogen, Groningen, Niederlande) elektrophoretisch aufgetrennt, welche anschließend einer Silberfärbung unterzogen wurden.

\section{Statistische Auswertung}

Um die im Stad 1-Verbund eingesetzten Mehl- bzw. Latex-HT-Lösungen exakt beurteilen zu können, wurden die Daten statistisch ausgewertet. Die Pricktestung bei 125 Bäckern bzw. 21 Beschäftigten im Gesundheitswesen resultierte in 250 bzw. 42 Tests pro Allergen, die in die Auswertung eingingen. Als Gold-Standard diente zunächst die sIgE-Bestimmung, da diese Daten für jeden Probanden verfügbar waren.

Mit Hilfe von Vierfeldertafeln wurden Testeffizienz [(rp $+\mathrm{rn}) /(\mathrm{rp}$ $+f p+r n+f n)]$, Sensitivität $[r p /(r p+f n)]$, Spezifität $[r n /(r n+f p)]$, positiver (PPV) $[\mathrm{rp} /(\mathrm{rp}+\mathrm{fp})]$ und negativer $[\mathrm{rn} /(\mathrm{rn}+\mathrm{fn})]$ prädiktiver Wert (NPV) bestimmt. Richtig Positive (rp) sind Personen mit positivem sIgE und positivem HT; richtig Negative (rn) sind Personen mit negativem sIgE und negativem HT; falsch Positive (fp) sind Personen mit negativem slgE und positivem HT; falsch Negative (fn) sind Personen mit positivem sIgE und negativem HT.

Da in einigen Fällen das Ergebnis eines spezifischen Provokationstests vorlag, erfolgte die Bewertung der Mehl- und LatexHT-Lösungen anschließend auch bezogen auf diesen Test als Gold-Standard.

Die statistische Analyse erfolgte unter Verwendung der SAS/STAT software, version 9.1 (Cary, N.C., U.S.; SAS, 2005).

\section{Ergebnisse \\ $\nabla$}

Ein Teil der Ergebnisse der Bewertungen der Mehl-HT-Lösungen wurde bereits publiziert [10].

\section{Protein- und Antigenbestimmungen, SDS-Gelektrophorese}

Zunächst wurde in vitro in allen vorliegenden HT-Lösungen der Protein- und Antigengehalt (für die Latex-HT-Lösungen der Allergengehalt) gemessen ( $\bullet$ Tab. 1). Dabei zeigten sich für alle Allergene (Weizen- und Roggenmehl, Latex, Tyrophagus putrescentiae) zum Teil deutliche Unterschiede zwischen den HT-Lösungen von verschiedenen Herstellern. Diese Diskrepanzen waren auch in der SDS-Gelektrophorese erkennbar $(\boldsymbol{\otimes}$ Abb. 1).

\section{slgE}

Von 125 Bäckern wiesen 85 (68\%) slgE gegen Weizenmehl (Bereich $0,35->100 \mathrm{kU} / \mathrm{L}$, Median 2,31 kU/L) und $83(66 \%)$ gegen Roggenmehl (Bereich 0,35->100 kU/L, Median 3,24 kU/L) auf. Bei acht der 21 Latex-Exponierten waren latexspezifische IgEAntikörper nachweisbar (Range 0,35-25,7 kU/L, Median $0,23 \mathrm{kU} / \mathrm{L}$ ). Beide Landwirte wiesen sIgE gegen Tyrophagus putrescentiae auf (8,47 bzw. 2,08 kU/L).

\section{Haut-Pricktest}

Mehl und Latex

Bei der Bewertung der HT-Lösungen für Mehle und Latex, die im Rahmen des Verbundprojektes Stad 1 an größeren Kollektiven getestet werden konnten, wurde zunächst überprüft, bei wel- 

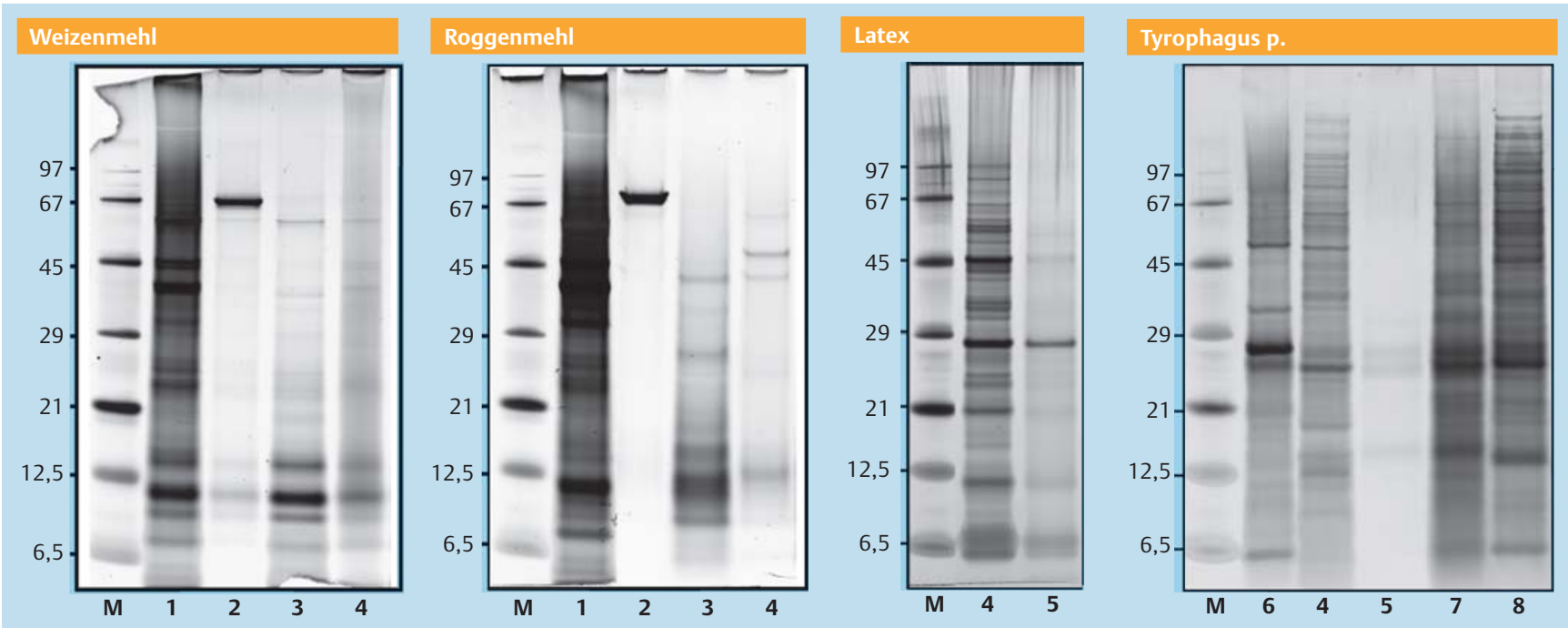

Abb. 1 Gleiche Volumina der verschiedenen HT-Lösungen mit Silber angefärbt nach Auftrennung im SDS-Gel (Hersteller: 1 IPA, 2 Hal, 3 Bencard, 4 Allergopharma, 5 Stallergènes, 6 Allerbio, 7 Lofarma, 8 ALK-Abelló).

Tab. 1 Protein- und Antigen-(Allergen-)gehalte verschiedener HT-Lösungen für drei ausgewählte Berufsallergene.

\begin{tabular}{|c|c|c|}
\hline HT-Lösung & $\begin{array}{l}\text { Protein } \\
(\mathrm{mg} / \mathrm{ml})\end{array}$ & $\begin{array}{l}\text { Antigen }(\mathrm{U} / \mathrm{ml}) \text { bzw. } \\
\text { Latexallergen }(\mu \mathrm{g} / \mathrm{ml})\end{array}$ \\
\hline \multicolumn{3}{|l|}{ Mehle } \\
\hline WM Hal & 237 & 49 \\
\hline WM Allergopharma & 191 & 158 \\
\hline WM Bencard & 451 & 501 \\
\hline WM IPA & 1827 & 945 \\
\hline RM Hal & 223 & 11 \\
\hline RM Allergopharma & 183 & 23 \\
\hline RM Bencard & 402 & 248 \\
\hline RM IPA & 2176 & 4766 \\
\hline \multicolumn{3}{|c|}{ Tyrophagus putrescentiae } \\
\hline Allerbio & 117 & 103 \\
\hline Allergopharma & 103 & 14 \\
\hline Stallergènes & u. N. & 31 \\
\hline Lofarma & 282 & 7 \\
\hline ALK-Abelló & 355 & 787 \\
\hline \multicolumn{3}{|l|}{ Latex } \\
\hline Allergopharma & 215 & 163 \\
\hline Stallergènes & u. N. & 16 \\
\hline
\end{tabular}

chem Cut-off-Wert (als positiv definierte Quaddelgröße), bezogen auf den Gold-Standard sIgE, die höchste Testeffizienz erreicht wurde. Es zeigte sich, dass dies für alle getesteten Mehl- und Latex-HT-Lösungen bereits bei einer Quaddelgröße $\geq 1,5 \mathrm{~mm}$ der Fall war ( $\bullet$ Tab. 2). Dabei lagen die Testeffizienzen der MehlHT-Lösungen zwischen $55 \%$ und $89 \%$, die der beiden Latex-HTLösungen jeweils bei $95 \%$. Alle weiteren Auswertungen wurden mit dem optimalen Cut-off $(\geq 1,5 \mathrm{~mm})$ durchgeführt.

Mit den verschiedenen Mehl-HT-Lösungen ergaben sich divergente Anzahlen positiver HT-Ergebnisse im Bäckerkollektiv (৫ Tab. 3). Bei 61-91\% der Doppelbestimmungen mit einem positiven Ergebnis war auch der zweite Test positiv. Im Gegensatz dazu führten die beiden Latex-HT-Lösungen im Kollektiv der Latex-Exponierten zu einer identischen Anzahl positiver HTs und zu einer 100\%igen Übereinstimmung der Ergebnisse im Rahmen der Doppelbestimmungen $(\bullet$ Tab. $\mathbf{3})$.
Tab. 2 Testeffizienz der verschiedenen HT-Lösungen für Weizenmehl (WM) und Roggenmehl (RM) bzw. Latex bei 125 Bäckern bzw. 21 Latex-Exponierten, bezogen auf den Gold-Standard slgE in Abhängigkeit vom Cut-off.

\begin{tabular}{|c|c|c|c|}
\hline \multirow[t]{2}{*}{ HT-Lösung } & \multicolumn{3}{|c|}{ Testeffizienz bei Cut-off } \\
\hline & $\geq 1,5 \mathrm{~mm}$ & $\geq 2,0 \mathrm{~mm}$ & $\geq 2,5 \mathrm{~mm}$ \\
\hline \multicolumn{4}{|l|}{ Mehle } \\
\hline WM Hal & $56,4 \%$ & $55,6 \%$ & $54,4 \%$ \\
\hline WM Allergopharma & $70,0 \%$ & $67,2 \%$ & $65,6 \%$ \\
\hline WM Bencard & $80,0 \%$ & $77,6 \%$ & $75,2 \%$ \\
\hline WM IPA & $86,8 \%$ & $86,0 \%$ & $82,8 \%$ \\
\hline RM Hal & $55,2 \%$ & $54,4 \%$ & $51,6 \%$ \\
\hline RM Allergopharma & $70,4 \%$ & $69,2 \%$ & $64,8 \%$ \\
\hline RM Bencard & $75,2 \%$ & $74,0 \%$ & $73,6 \%$ \\
\hline RM IPA & $89,2 \%$ & $88,4 \%$ & $85,6 \%$ \\
\hline \multicolumn{4}{|l|}{ Latex } \\
\hline Allergopharma & $95,2 \%$ & $95,2 \%$ & $95,2 \%$ \\
\hline Stallergènes & $95,2 \%$ & $95,2 \%$ & $90,5 \%$ \\
\hline
\end{tabular}

Tab.3 Übereinstimmung der Doppelbestimmungen mit verschiedenen HT-Lösungen für Weizenmehl (WM) und Roggenmehl (RM) bzw. Latex bei 125 Bäckern bzw. 21 Latex-Exponierten (Cut-off $\geq 1,5 \mathrm{~mm}$ ).

\begin{tabular}{|c|c|c|c|}
\hline HT-Lösung & $\begin{array}{l}\text { Mind. eine } \\
\text { Quaddel } \\
\text { positiv (n) }\end{array}$ & $\begin{array}{l}\text { Beide } \\
\text { Quaddeln } \\
\text { positiv (n) }\end{array}$ & $\begin{array}{l}\text { Überein- } \\
\text { stimmung }\end{array}$ \\
\hline \multicolumn{4}{|l|}{ Mehle } \\
\hline WM Hal & 42 & 26 & $62 \%$ \\
\hline WM Allergopharma & 61 & 48 & $79 \%$ \\
\hline WM Bencard & 78 & 64 & $82 \%$ \\
\hline WM IPA & 87 & 76 & $87 \%$ \\
\hline RM Hal & 36 & 22 & $61 \%$ \\
\hline RM Allergopharma & 64 & 39 & $61 \%$ \\
\hline RM Bencard & 70 & 58 & $83 \%$ \\
\hline RM IPA & 93 & 85 & $91 \%$ \\
\hline \multicolumn{4}{|l|}{ Latex } \\
\hline Allergopharma & 9 & 9 & $100 \%$ \\
\hline Stallergènes & 9 & 9 & $100 \%$ \\
\hline
\end{tabular}


Tab. 4 Bewertung der verschiedenen HT-Lösungen für Weizenmehl (WM) und Roggenmehl (RM) bzw. Latex bei 125 Bäckern bzw. 21 Latex-Exponierten (Cut-off $\geq 1,5 \mathrm{~mm}$ ), bezogen auf den Gold-Standard slgE.

\begin{tabular}{|c|c|c|c|c|}
\hline HT-Lösung & Sensitivität & Spezifität & PPV & NPV \\
\hline \multicolumn{5}{|l|}{ Mehle } \\
\hline WM Hal & $38 \%$ & $95 \%$ & $94 \%$ & $42 \%$ \\
\hline WM Allergopharma & $60 \%$ & $91 \%$ & $94 \%$ & $52 \%$ \\
\hline WM Bencard & $77 \%$ & $86 \%$ & $92 \%$ & $64 \%$ \\
\hline WM IPA & $88 \%$ & $84 \%$ & $92 \%$ & $77 \%$ \\
\hline RM Hal & $34 \%$ & $98 \%$ & $97 \%$ & $43 \%$ \\
\hline RM Allergopharma & $58 \%$ & $94 \%$ & $95 \%$ & $53 \%$ \\
\hline RM Bencard & $70 \%$ & $86 \%$ & $91 \%$ & $59 \%$ \\
\hline RM IPA & $95 \%$ & $77 \%$ & $89 \%$ & $89 \%$ \\
\hline \multicolumn{5}{|l|}{ Latex } \\
\hline Allergopharma & $100 \%$ & $92 \%$ & $88 \%$ & $100 \%$ \\
\hline Stallergènes & $100 \%$ & $92 \%$ & $88 \%$ & $100 \%$ \\
\hline
\end{tabular}

Ebenfalls beim optimalen Cut-off-Wert und wiederum bezogen auf den Gold-Standard sIgE wurden neben der Testeffizienz $(\checkmark$ Tab. 2), Sensitivität, Spezifität, PPV und NPV der Mehl- und Latex-HT-Lösungen ermittelt ( Tab. 4). Während für die beiden Latex-HT-Lösungen alle Werte identisch waren, variierten bei den verschiedenen Mehl-HT-Lösungen insbesondere Sensitivität und NPV sehr stark. Dabei sind die Unterschiede zwischen Weizen- und Roggenmehl klein, die zwischen den verschiedenen Herstellern jedoch groß.

Von 95 Bäckern, die sich einem spezifischen Provokationstest mit Roggenmehl unterzogen hatten, reagierten 63 (66\%) positiv. Eine vergleichende Bewertung der Mehl-HT-Lösungen in diesem Subkollektiv ( $n=95$ ), zum einen bezogen auf den Gold-Standard sIgE und zum anderen bezogen auf den Gold-Standard Provokation, ergab nur unwesentliche Unterschiede [10]. Von den zehn Latex-Exponierten, die sich einem Handschuhtragetest unterzogen hatten, zeigten sechs (60\%) eine positive Reaktion in diesem Test. Auch hier unterscheidet sich die Bewertung der HT-Lösungen je nach Gold-Standard nur in geringem Maße $($ Abb. 2$)$.

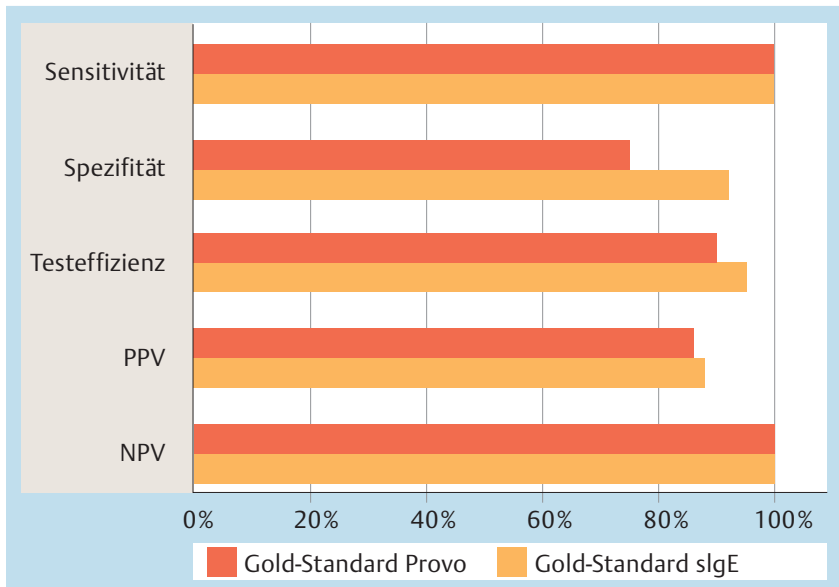

Abb. 2 Sensitivität, Spezifität, Testeffizienz sowie positiver (PPV) und negativer prädiktiver Wert (NPV) der Latex-HT-Lösungen beim optimalen Cutoff von $\geq 1,5 \mathrm{~mm}$, bezogen auf den Gold-Standard „Latex-slgE“ bzw. „spezifischer Provokationstest“" $(n=10)$. Beide Latex-HT-Lösungen lieferten identische Werte.

\section{Tyrophagus putrescentiae}

Da die verschiedenen HT-Lösungen für die Vorratsmilbe Tyrophagus putrescentiae nicht im Rahmen des Verbundprojektes Stad 1 eingesetzt worden waren, wurden bisher erst zwei symptomatische Landwirte mit sIgE gegen diese Vorratsmilbe im titrierten Pricktest untersucht. Dabei war das Ergebnis von der verwendeten HT-Lösung sowie vom Sensibilisierungsgrad (sIgE-Titer) der Patienten abhängig ( $\bullet$ Abb. 3). Bei beiden Landwirten verlief der spezifische Provokationstest mit Tyrophagus putrescentiae positiv.

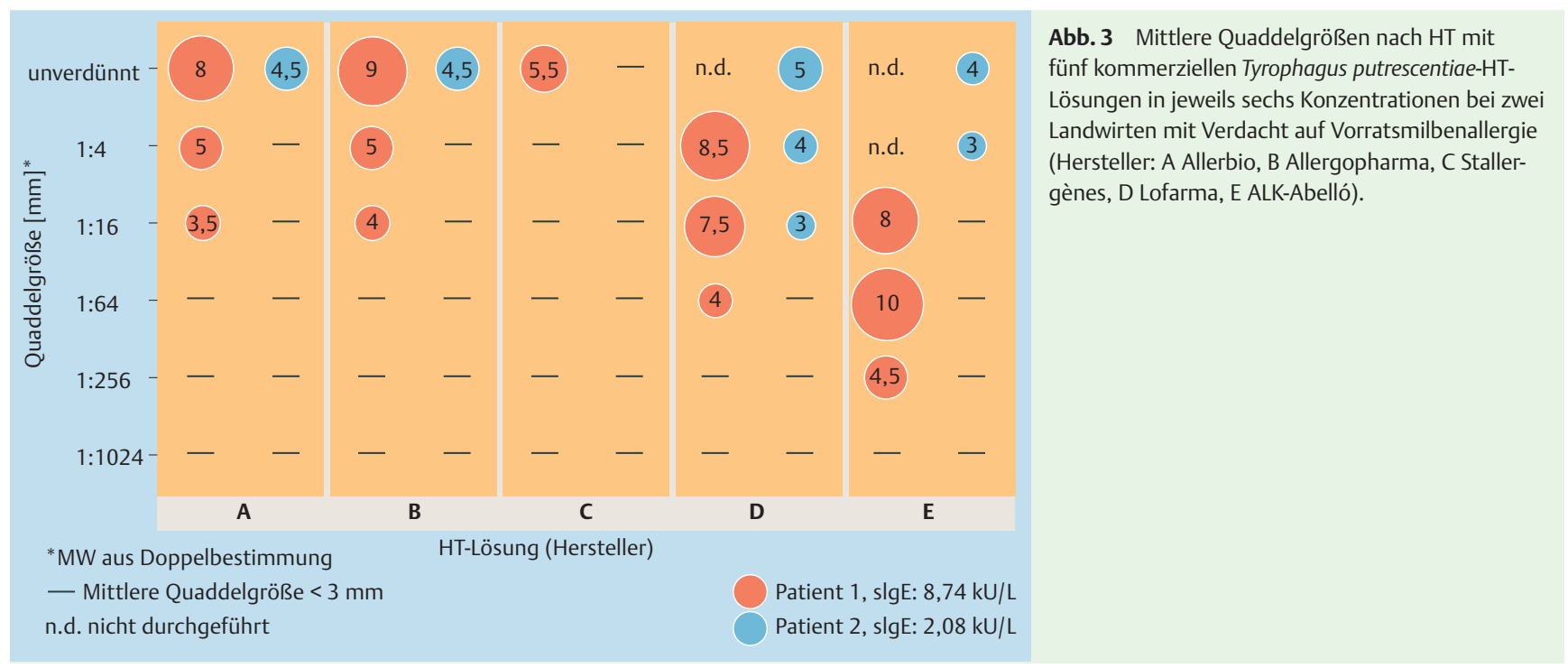




\section{Diskussion}

$\nabla$

Bei der Diagnostik von Typ-I-Allergien gilt der HT als einer der zentralen Bausteine. Er ist wenig invasiv, relativ leicht zu quantifizieren und korreliert in der Regel gut mit entsprechenden Organprovokationen [13]. Hinzu kommt, dass der HT finanziell günstiger ist als viele andere diagnostische Tests [14] und das Ergebnis unmittelbar zur Verfügung steht. Auch für die Beurteilung berufsbedingter Sensibilisierungen ist der HT wichtig; so hat er eine große Relevanz für die Begutachtung und die damit verbundenen sozioökonomischen Konsequenzen. Aus diesem Grunde wurde die Qualität von HT-Lösungen für ausgewählte Berufsallergene zunächst in vitro und dann in vivo verglichen und beurteilt.

Für alle untersuchten Berufsallergene (Mehle, Latex, Vorratsmilbe) zeigten die HT-Lösungen in der SDS-Gelelektrophorese in Abhängigkeit vom Hersteller zum Teil große Unterschiede hinsichtlich Anzahl und Intensität der Proteinbanden. Auch die Ergebnisse der Protein- und Antigen-(Allergen-)Quantifizierungen variierten teilweise stark.

Trotz dieser Unterschiede ergaben beide Latex-HT-Lösungen in der parallelen Hauttestung übereinstimmend eine maximale Sensitivität bei guter Spezifität (92\%). Dies ist vermutlich dadurch zu erklären, dass trotz der unterschiedlichen Mengen an Gesamtprotein und -allergen in beiden untersuchten Latex-HTLösungen die für die Diagnostik relevanten Hauptallergene in ausreichender Menge vorhanden sind. Für Beschäftigte im Gesundheitswesen sind dies in der Regel die Allergene Hev b 2, 5 , 6.01 und 13 [15].

Vergleichende Bewertungen von HT-Lösungen verschiedener Hersteller, insbesondere für Berufsallergene, sind in der Literatur bisher relativ selten beschrieben. In einer Studie wurden LatexHT-Lösungen von drei verschiedenen Herstellern getestet, allerdings in einem Kollektiv von 46 Kindern mit Verdacht auf eine Latexallergie. Der ebenfalls im Rahmen der vorliegenden Publikation getestete Extrakt (Stallergenès) erreichte dabei eine relativ gute Sensitivität ( $80 \%$ ) bei einer Spezifität von $88 \%$, die Extrakte der anderen beiden Hersteller Sensitivitäten von 65\% bzw. 96\% bei einer Spezifität von 94\% [16]. Bei dem Vergleich der Ergebnisse muss berücksichtigt werden, dass die Tests in der beschriebenen Studie nicht an beruflich sensibilisierten Erwachsenen, sondern an Kindern durchgeführt wurden, die die Latexallergie in der Regel durch häufige Operationen erworben hatten und für deren Sensibilisierung in der Regel andere Allergene (Hev b 1 und 3 [15]) bedeutsam sind.

Im Gegensatz zu den Latex-HT-Lösungen, ergab die In-vivo-Testung der verschiedenen Mehl-HT-Lösungen deutliche Unterschiede, insbesondere hinsichtlich Sensitivität, Testeffizienz und NPV. Dabei zeigten in der Regel Mehl-HT-Lösungen mit höherem Protein- und Antigengehalt bzw. einer höheren Anzahl von Proteinbanden im SDS-Gel höhere Werte.

Auch Unterschiede in der Qualität von Mehl-HT-Lösungen anderer Hersteller wurden in einem kleineren Kollektiv bereits früher beschrieben [1].

Die statistische Auswertung der HT-Ergebnisse mit Mehlen und Latex ermöglichte die Ermittlung der Quaddelgröße, bei der die maximale Testeffizienz der HT-Lösungen erreicht wurde (optimaler Cut-off). In allen Fällen war dies bereits bei sehr kleinen Quaddelgrößen $(\geq 1,5 \mathrm{~mm})$ der Fall. Während in der medizinischen Routine in den meisten Fällen Quaddeln $\geq 3 \mathrm{~mm}$ als positiv gelten, ergab die Testung mit HT-Lösungen für Hausstaubmilbe, Katze, Lieschgras und Cladosporium in einer Studie mit mehr als
11000 Probanden, dass in epidemiologischen Studien ein Positivkriterium einer Quaddel $>0 \mathrm{~mm}$ am besten geeignet ist, um allergische Sensibilisierungen zu erfassen [17].

Da zudem die Diagnostik in der arbeitsmedizinischen Begutachtung in der Regel mehrstufig verläuft (Anamnese, Pricktest, slgEBestimmung, Provokationstestung), erscheint eine hohe Sensitivität der diagnostischen Tests von großer Bedeutung.

In diesem Zusammenhang muss auch berücksichtigt werden, dass die Übereinstimmung der HT-Doppelbestimmungen bei Bäckern mit mindestens einem positiven Test von der jeweiligen Mehl-HT-Lösung abhängig war. Da die Reproduzierbarkeit der HT-Ergebnisse nicht immer optimal war, sind beim HT Mehrfach-, mindestens jedoch Doppelbestimmungen sinnvoll. Dies wird auch von anderen Autoren gefordert [4]. Insbesondere bei schwach sensibilisierten Patienten besteht die Gefahr, dass der HT falsch negativ verläuft, weil z.B. die Haut nicht ausreichend eingeritzt wird und somit nicht genügend Allergen eindringt.

Mit beiden Gold-Standards (sIgE oder spezifischer Provokationstest) ergaben sich sowohl für die Beurteilung der Mehl- [10] als auch der Latex-Hauttestlösungen ähnliche Werte.

Da die Testungen mit Tyrophagus putrescentiae bisher nur in zwei Probanden durchgeführt wurden, konnte der optimale Cut-off bisher nicht ermittelt werden; deshalb wurden Quaddeln von $3 \mathrm{~mm}$ oder mehr als positiv angesehen. Dies scheint angemessen vor dem Hintergrund, dass Kanceljak-Macan u. Mitarb. [18] nach Pricktestung mit HT-Lösungen für Tyrophagus putrescentiae (ALK-Abeló) in 457 Personen schlussfolgern, dass für die klinische Beurteilung des Tests ein Cut-off-Wert von 4,5 mm aufgrund der niedrigen Spezifität zu bevorzugen ist.

In vivo ergaben die verschiedenen HT-Lösungen für Tyrophagus putrescentiae unterschiedliche Ergebnisse. So führte die HT-Lösung mit dem geringsten Proteingehalt bei einem der Landwirte, der über arbeitsplatzbezogene Beschwerden klagte und sowohl im IgE- als auch im bronchialen Provokationstest auf Tyrophagus putrescentiae positiv reagierte, zu einem falsch-negativen Ergebnis.

Die In-vitro- und In-vivo-Testung von HT-Lösungen für ausgewählte Berufsallergene zeigte für Mehle und Tyrophagus putrescentiae deutliche Unterschiede zwischen den HT-Lösungen verschiedener Hersteller. Vor allem die detaillierte Bewertung der HT-Ergebnisse bei 125 Bäckern mit verschiedenen Weizen- und Roggenmehl-HT-Lösungen ergab, dass die Qualität einzelner HT-Lösungen für eine zuverlässige Diagnostik nicht ausreichend war. Erste Untersuchungen mit HT-Lösungen für Tyrophagus putrescentiae verschiedener Hersteller wiesen ebenfalls auf qualitative Unterschiede in den verschiedenen kommerziellen Lösungen hin. Dabei wiesen HT-Lösungen mit höheren Protein- bzw. Antigengehalten eine bessere Sensitivität auf.

Um zu klären, wie die Qualität von HT-Lösungen für andere Berufsallergene zu beurteilen ist, werden derzeit in einer europäischen Multicenterstudie HT-Lösungen für weitere Mehle, Mehlschädlinge, Enzyme, Latex und kreuzreagierende Nahrungsmittel, Getreidestäube, Vorratsmilben, Schimmelpilze, Nutztiere sowie Labortiere in möglichst großen Kollektiven im vergleichenden HT bei entsprechend exponierten symptomatischen Personen getestet. Durch die anschließende Bewertung, welche HT-Lösungen für die Diagnostik der entsprechenden beruflichen Sensibilisierungen geeignet sind, soll vermieden werden, dass die Ergebnisse des Pricktests durch HT-Lösungen mit einer mangelhaften Qualität limitiert werden. 


\section{Danksagung}

Unser Dank gilt den Mitgliedern des Stad 1-Verbundes (H.-C. Broding, damals Institut und Poliklinik für Arbeits-, Sozial- und Umweltmedizin Erlangen; C. Keller, Pneumologische Praxis, Frankfurt/Main; H. Muesken, Pneumologische Praxis Bad Lippspringe; A. Overlack, Pneumologische Praxis Bonn; G. Schultze-Werninghaus, Berufsgenossenschaftliches Universitätsklinikum Bergmannsheil Bochum, J. Walusiak, Nofer Institute of Occupational Medicine Lodz, Polen) für die Untersuchung von Patienten im Rahmen des Verbundprojektes. Wir danken S. Rabstein für die Unterstützung bei der Auswertung der Daten sowie E. Zahradnik, C. Fleischer und U. Meurer für die ausgezeichneten Laborarbeiten.

\section{Interessenkonflikte}

Die Autoren geben an, dass kein Interessenkonflikt besteht.

\section{Literatur}

1 Sander I, Merget R, Degens PO et al. Comparison of wheat and rye flour skin prick test solutions for diagnosis of baker's asthma. Allergy 2004; 59: $95-98$

2 Deutsche Gesetzliche Unfallversicherung (DGUV), ed. Geschäfts- und Rechnungsergebnisse der gewerblichen Berufsgenossenschaften 2008. Sankt Augustin: 2009

3 Müsken H, Franz JT, Wahl R et al. Sensitization to different mite species in German farmers: in vitro analyses. J Investig Allergol Clin Immunol 2003; $13: 26-35$

4 Dreborg $S$. Methods for skin testing. EAACI Position paper. Allergy 1989; 44 (Suppl. 10): $22-30$

5 Dreborg S, Frew A. Allergen standardization and skin tests. EAACI Position paper. Allergy 1993; 48 (Suppl. 14): 49-82

6 Sander I, Kespohl S, Merget R et al. A new method to bind allergens for the measurement of specific IgE antibodies. Int Arch Allergy Immunol 2005; 136: $39-44$
7 van Kampen V, Rabstein S, Sander I et al. Prediction of challenge test results by flour-specific IgE and skin prick test in symptomatic bakers. Allergy 2008; 63: 897-902

8 Bradford MM. A rapid and sensitive method for the quantitation of microgram quantities of protein utilizing the principle of protein-dye binding. Anal Biochem 1976; 72 : 48-254

9 Bogdanovic J, Wouters IM, Sander I et al. Airborne exposure to wheat allergens: measurement by human immunoglobulin G4 and rabbit immunoglobulin G immunoassays. Clin Exp Allergy 2006; 36: 1168 1175

10 vanKampen $V$, Merget $R$, Rabstein $S$ et al. Comparison of wheat and rye flour solutions for skin prick testing: a multi-centre study (Stad 1). Clin Exp Allergy 2009; 39: 1896 - 1902

11 Zahradnik E, Sander I, Fleischer C et al. Entwicklung von Enzymimmunoassays zur Quantifizierung von Vorratsmilbenantigenen in arbeitsplatzbezogenen Staubproben. Gefahrstoffe- Reinhaltung der Luft 2009; 9: 369- 375

12 Baur X, Chen Z, Raulf-Heimsoth $M$ et al. Protein and allergen content of various natural latex articles. Allergy 1997; 52: 661 - 664

13 Oppenheimer J, Nelson HS. Skin testing. Ann Allergy Asthma Immunol 2006; 96 (Suppl. 1): 6-12

14 Borghesan F, Bernardi $D$, Plebani $M$. In vivo and in vitro allergy diagnostics: it's time to re-appraise the costs. Clin Chem Lab Med 2007; 45: $391-395$

15 Raulf-Heimsoth M, Rihs HP, Rozynek P et al. Quantitative analysis of immunoglobulin $\mathrm{E}$ reactivity profiles in patients allergic or sensitized to natural rubber latex (Hevea brasiliensis). Clin Exp Allergy 2007; 37: $1657-1667$

16 Bernardini R, Pucci N, Azzari C et al. Sensitivity and specificity of different skin prick tests with latex extracts in pediatric patients with suspected natural rubber latex allergy-a cohort study. Pediatr Allergy Immunol 2008; 19: 315-318

17 Bousquet PJ, Chatzi L, Jarvis D et al. Assessing skin prick tests reliability in ECRHS-I. Allergy 2008; 63: 341 - 346

18 Kanceljak-Macan B, Macan J, Plavec $D$ et al. The $3 \mathrm{~mm}$ skin prick test (SPT) threshold criterion is not reliable for Tyrophagus putrescentiae: the re-evaluation of SPT criterion to dust mites. Allergy 2002; 57: $187-1190$ 